\title{
How do citizens perceive road safety and mobility? The role of opinion surveys and examples from the ROSEE project
}

\author{
S. Rossetti \& M. Tiboni \\ DICATAM, Università degli Studi di Brescia, Italy
}

\begin{abstract}
Urban and territorial planning should strive to increase the quality of citizens' lives. Therefore, planners cannot avoid to think in terms of sustainability, trying to provide high levels of quality of life not only to the current, but to the future generations alike. Road safety is a fundamental requirement of a sustainable city, but road accidents still represent a significant public health and economic problem. Within this framework, this paper will focus on the importance of human perception while dealing with urban planning, and in particular, with mobility and road safety.

Indeed, collecting road accident data is not enough to completely understand the risk dynamics that take place every day on our roads: listening to the road users and to their opinions may be helpful to better assess road safety related threats, and to therefore plan safer and more sustainable cities. The aim of the proposed paper is to demonstrate that, in order to properly plan interventions in the field of road safety, road accident data must be highly integrated with road users' opinions that can be investigated through opinion surveys.

This paper will present the methodologies and the results of opinion surveys that were conducted in the north of Italy during the period 2011-2013, to investigate the behaviour and perceptions of different road users, with different levels of vulnerability.
\end{abstract}

Keywords: road safety, opinion surveys, ROSEE. 


\section{Opinion surveys as complementary approaches to road accident data analysis}

Urban and territorial planning should strive to increase the quality of citizens' lives. Therefore, planners cannot avoid to think in terms of sustainability, trying to provide high levels of quality of life not only to the current, but to the future generations alike. Road safety is a fundamental requirement of a sustainable city, but road accidents still represent a significant public health and economic problem (see Busi [1]; Tira [2]; Tiboni [3]). Within this framework, the present paper focuses on the importance of human perception while dealing with urban planning, and in particular, with mobility and road safety.

Data on road accidents in urban areas is of crucial importance while dealing and planning for road safety (see Fleury [4]; Tiboni and Rossetti [5]). Nowadays, the collection and analysis of accident data has become common practice in Europe, and has reached a good level of quality. In Italy, the National Institute for Statistics (ISTAT) and the Automobile Club (ACI) publish road accident statistics every year, based on police records. Furthermore, there are many international databases and information sources that gather and disseminate road safety related data, such as the European Commission Database of Road Accidents (CARE), the European Road Safety Observatory (ERSO), the International Road Traffic Accident Database (IRTAD), and the Statistical Office of the European Union (EUROSTAT).

However, collecting data is not enough to completely understand the risk dynamics that take place every day on our roads. Therefore, listening to the users and to their opinions may be helpful to better assess road safety related threats. The aim of this paper is to demonstrate that, in order to properly plan interventions in the field of road safety, road accident data must be highly integrated with road users' opinions that can be investigated through opinion surveys.

Opinion surveys represent a statistical research method, quite common in the field of Social Sciences, such as psychology, sociology, and communication studies. Surveys, in the broadest sense, aim at the "collection of quantified data from a population for purposes of description, or to identify covariation between variables that may point to causal relationships or predictive patterns of influence" (Sapsford [6]). The purpose of a survey is to produce quantitative or numerical descriptions of some aspects of the study population (Fowler [7]). Literature on the survey methodology for the Social Sciences is presently very wide, covering many aspects of conducting surveys, including sampling, measuring, validating and reporting issues.

In the field of Engineering Sciences, public surveys are often used in transport and mobility management. For example, surveys are a frequently used tool in O/D (Origin/Destination) studies, to conduct a review of travel information, or to investigate travellers' attitudes (see Pronello [8]).

Furthermore, questionnaires that investigate home to work movements are always applied in the diagnostic phase of Travel Plans. A Travel Plan, which in Italy is known as "Piano degli spostamenti casa-lavoro (PSCL)", is a package of measures aimed at promoting sustainable travel in an organisation (school, 
business, public body, etc.). The goals of Travel Plans are to discourage car use (particularly single occupant), encourage walking, cycling and public transport use, and provide safe access to all users. A Travel Plan can bring different benefits to the organisation, including a reduction of parking and congestion problems near the site, an improvement of a company's image and green credentials, and an improvement of employees' health conditions. Furthermore, the production of Travel Plans can be an opportunity to introduce road safety into the culture of an organisation.

The mobility manager of a company, who is the person in charge of developing the Travel Plan, should arrange a questionnaire, and may prepare a questionnaire to be completed by staff, customers and suppliers in order to investigate their existing travel patterns, their aspirations for the future, and the barriers to them making changes.

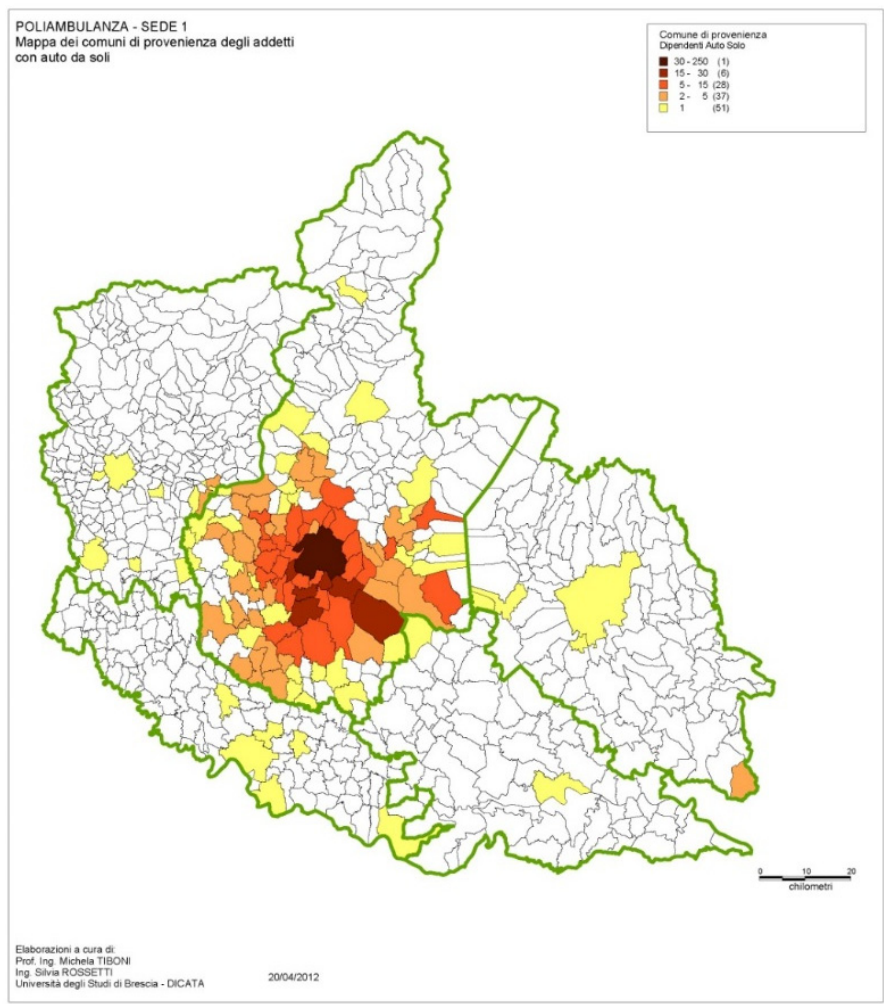

Figure 1: Elaboration of a survey carried out in a hospital in Brescia (Poliambulanza) during the diagnostic phase of its Travel Plan (April 2012). This map highlights the municipalities of origin for the employees that usually arrive to their work site by car as single occupants. 
Public surveys can also be applied to investigate road safety attitudes (e.g. alcohol impaired driving, overspeeding, etc.), even though their use is in this case less common. In psychology, the simplest definition of attitude is the judgment human beings make of everything around them (their environment, other people, situations, and themselves) as good or bad, favourable or unfavourable, positive or negative, pleasurable or displeasing, likeable or unlikeable (Albarracin et al. [9]).

In recent years, some countries have started to carry out surveys focused on road users' attitudes and behaviour.

To provide a good practice example, one can refer to the New Zealand survey on public attitudes to road safety (New Zealand Ministry of Transport [10]). This survey has been undertaken periodically since 1974 and annually since 1994 to evaluate attitudes to road safety issues. It focuses on alcohol consumption, over speeding and the use of safety belts. In addition, respondents are asked to give their views on more general road safety issues.

In the UK, within the public awareness campaign on road safety THINK!, run by the Department for Transport, an annual research survey has been conducted since 2006 to assess road safety attitudes and behaviour among the British population.

At the European level, the European Commission co-financed a project entitled SARTRE "Social Attitudes to Road Safety Risk in Europe". The objective of the project was to survey, with a uniform methodology, attitudes, opinions, selfreported behaviour and experiences of European drivers, passengers and road users.

But nowadays, in Italy there is still a lack of detailed information on road users' attitudes: certified statistics on road safety attitudes and behaviour (e.g. alcohol impaired driving, safety belts and child restraint systems use...) are only scarcely available. Furthermore, the use of public opinion surveys to investigate not only road safety attitudes and behaviour, but in addition also the road users' perception of safety risks is even less common. For all these reasons, some pilot surveys, are at this point presented.

\section{Applying opinion surveys to investigate road users' perceptions}

This section deals with the methodologies and results of opinion surveys that have been conducted in Brescia and in the surrounding Provinces during the period of 2011-2013, to investigate the perception of road safety and accessibility issues that different road users, more or less vulnerable, have while thinking about their daily movements. 


\subsection{SOL assessment of public knowledge and opinion survey}

The first survey presented here was conducted in June 2011, within the framework of the SOL (Save Our Lives) European project and in cooperation with the University of Brescia, DICATAM research group, ALOT s.c.a r.l., and the Polish Motor Transport Institute with the Motor Transport Institute of Warsaw (ITS).

The survey was addressed to a general public, and implemented by means of an on-line questionnaire, aimed to reach as many community members as possible. The survey was composed of 26 questions. It was focused on the transportation habits of the interviewees and on their road safety and risk perceptions, for example, to investigate which are the most common mobility patterns in the community; why people tend to prefer or avoid a certain transportation mode (e.g. public bus, private vehicle); which are the main road safety attitudes such as the perception of the risks of a road crash as a pedestrian or cyclist in comparison to other risks in the community; and which are the biggest risks in transport according to the respondents' perceptions.

In the East Lombardy Region (Provinces of Brescia, Bergamo, Cremona and Mantova), 459 filled-in questionnaires were collected and analysed. The average respondent was 35.59 years old, had a university degree $(69.5 \%)$ and a driving license $(96.3 \%)$ since an average age of 16.11 years.

What emerged from the survey?

In the selected region of Italy a significant number of inhabitants get to work/school by car (67\%), and a similar number of respondents take advantage of other possibilities of transport (over 10\%), with the exception of motorcycles, which are used by a small number of survey participants. The inhabitants of the region have the longest distance to work/school $(20 \mathrm{~km})$, and the shortest one to main public offices (approx. $3 \mathrm{~km}$ ). Other destinations can be reached by them within 4-5 km.

All of the scores provided for assessment of the public transport are above average. The best score relates to safety, the worst one to comfortable working hours. The safety of roads in the region was evaluated above the average. The respondents believe that the factor that contributes the most to safety on roads is the usage of child restraint systems, and the factors that have the smallest influence are speed cameras and existing speed limits. However, all of the factors included in the questionnaire were perceived as significant (above average).

The majority of respondents claim that it is necessary to intensify publicity and advertising regarding road safety issues, as well as to increase police enforcement. At the same time, many survey participants believe that penalties for breaking road safety laws should remain at the same level. According to the respondents, road safety mainly depends on the behaviour and culture of road users, and on education and training. Nevertheless, other factors also seem to be important to them.

Questions concerning the perception of the road situation showed that the respondents have a low sense of control over what is happening on the road, and it often depends on luck, or the lack thereof.

Among the negative behaviour exhibited in the questionnaire, the respondents mainly indicate that they exceed speed limits, transport children without child 
restraint systems, and that they sometimes, although rarely, drive under the influence of alcohol.

The respondents evaluated the infrastructure in their region for pedestrians and bikers as average. The best score related to zebra crossings, the worst to bicycle racks/parking.

The survey participants are stopped by traffic control measures on an average of once a year. They also estimate the chances of being stopped for this reason as quite low.

The respondents will be more willing to walk if drivers were more careful and if streets had better lightning. Other factors were perceived as of average importance. Furthermore, they will be willing to cycle more if the number of bike lanes was higher.

It is important to draw attention to some of outcomes of this first survey, in particular related to the road users' perception of the road safety risks, and to the approaches they suggested to increase road safety.

On one hand, many respondents think that road safety highly depends on education and on the behaviour of the road users (figure 2), and that publicity and advertising regarding road safety issues and police enforcement should be increased to improve road safety (figure 3).

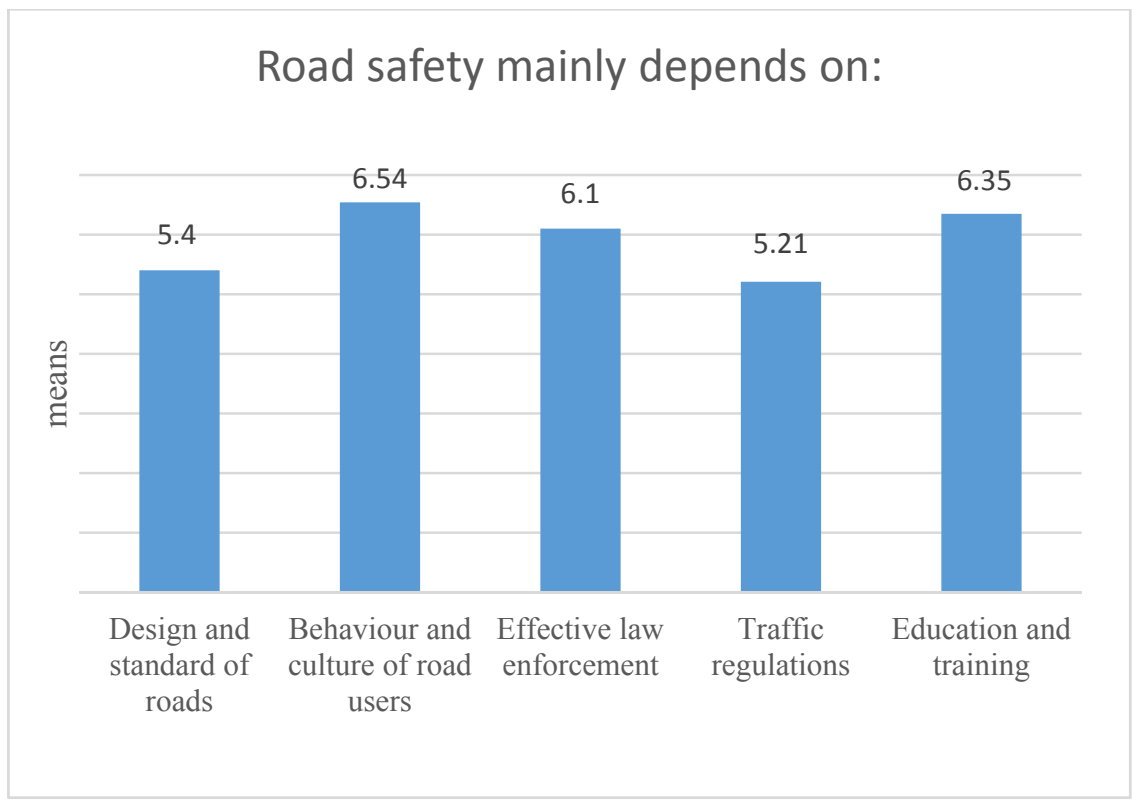

Figure 2: Respondents' perceptions of the dependence of some factors on road safety. 


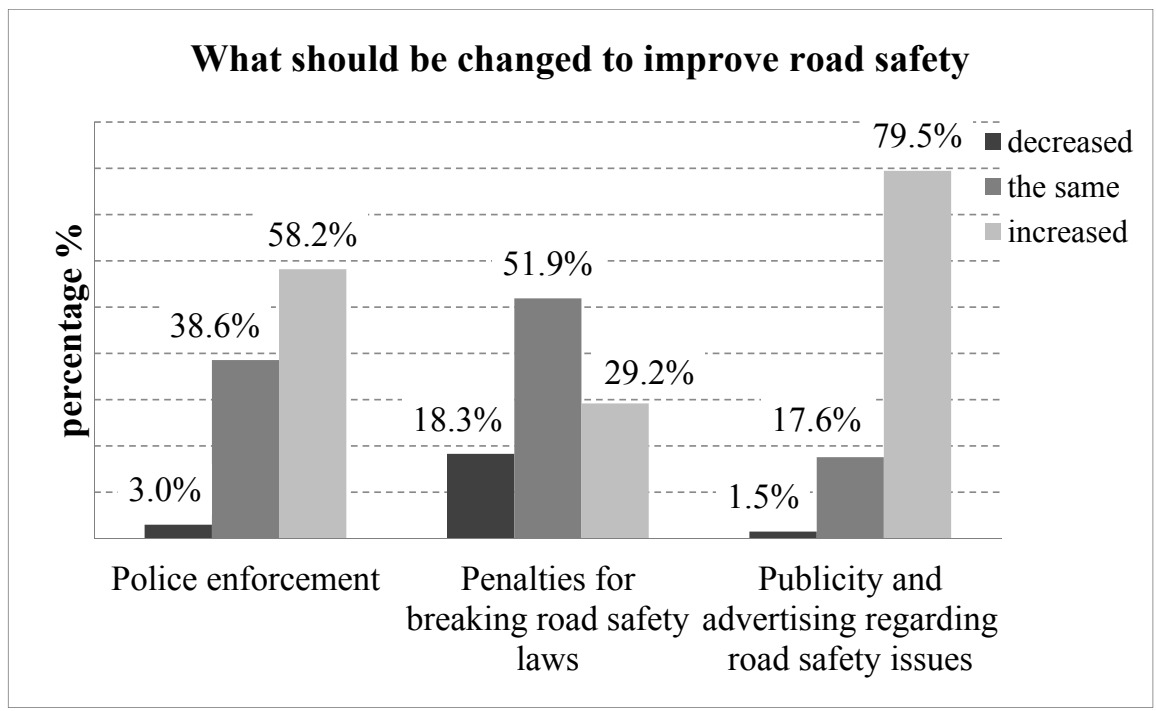

Figure 3: Respondents' perceptions of what should be changed to improve road safety.

On the other hand, it should be noted that the survey revealed a bit of road safety related to fatalism in the respondents' views: interviewee's opinions that fate and luck have a great influence on road accidents.

The respondents answered the question "to what extent do you agree with the following statements?" to a 7-point scale. The following table (table 1) includes the mean results: a higher score infers a higher acceptance of the statement.

Table 1: $\quad$ Perception of the road situation. Source: SOL public opinion survey, 2011.

\begin{tabular}{|l|c|}
\hline \multicolumn{1}{|c|}{ Statement } & M \\
\hline Dangerous situations on the road happen to us because of a bad luck & 3.11 \\
I often feel that I do not have influence on things that happen to me on & 5.12 \\
the road & 2.09 \\
I am responsible myself for things that happen to me on the road & 5.17 \\
Some people are just born unlucky and they are also unlucky on the road & \\
\hline
\end{tabular}

Table 1 shows that many people strongly agree with the sentence "Some people are just born unlucky and they are also unlucky on the road". This means that the population perceives road safety risks as a simply fact of luck. But, this is quite a false perception: people simply accept the risks of crashes, and there is a lack of awareness of the relationship that exists between infrastructures, vehicles, behaviour, and road safety. Starting from the results of this first assessment survey, we decided to proceed in the use of other surveys, and to further investigate the 
road users' perception of safety, and partially also of accessibility issues. Different categories were chosen, including students, professional drivers and road practitioners.

\subsection{The role of technicians (ROSEE STA questionnaire)}

Finally, what do professional engineers and stakeholders think about road safety?

Within the ROSEE (Road Safety in South East European regions) EU Project, the crucial role of surveys emerged once again, to assess demands and views of road safety stakeholders in each partner country. Thus, a stakeholder survey (STA questionnaire) was carried out, in cooperation with the National Technical University of Athens (NTUA) - Department of Transportation Planning and Engineering. The purpose of the questionnaire was to identify which data and tools are required by road safety stakeholders and technicians in partner countries, and to better understand the viewpoints of stakeholders who may not be directly involved in decision-making. The structure of the questionnaire has its roots in the framework of the DaCoTA (Road Safety Data, Collection, Transfer and Analysis) EU project, in which the National Technical University of Athens was partner. The questionnaire was divided into seven sections: country; field of work; use of tools; data and resources for fact finding and diagnosis of road safety issues; data and resources for the development of road safety related programmes; data and resources for the implementation of road safety related measures; data and resources for the monitoring and evaluation of road safety measures.

Concerning Italy, the DICATAM research group collected ten filled-in questionnaires from a selected group of stakeholders. The respondents, who were members of the Italian National Advisory Group (NAG) of the ROSEE project, were technicians (both from public administrations and from the private sector), researchers, university professors, and industry representatives. All of them deal with road safety issues for work, mainly on Data Collection and Analysis, Planning and Design, Communication, Monitoring and Evaluation, and Research (figure 4).

The interviewed stakeholders mainly consider their organisations as being quite or very influential on the public and on regional/local authorities, less influential or not influential at all on the National Government or on the European Commission. Eurostat, CARE and IRTAD databases are among the most used international information sources. National databases and information sources are frequently used as well, including national road accident databases, travel survey results and other exposure databases. All the respondents are moderately satisfied with the data and resources available to support their everyday activities.

The survey revealed that many data, definitions and studies, considered as having a high or medium priority for the respondents' work activities, are at the moment only partially or not available at all in Italy. These include a common definition of serious injury, data on the underreporting of road traffic crashes, crash databases that link police and hospital data, exposure data, information on the impacts of road safety measures on other sectors' policies and vice versa, standardised methods for carrying out evaluations of road safety measures, and information on the costs and benefits of a road safety measure. 


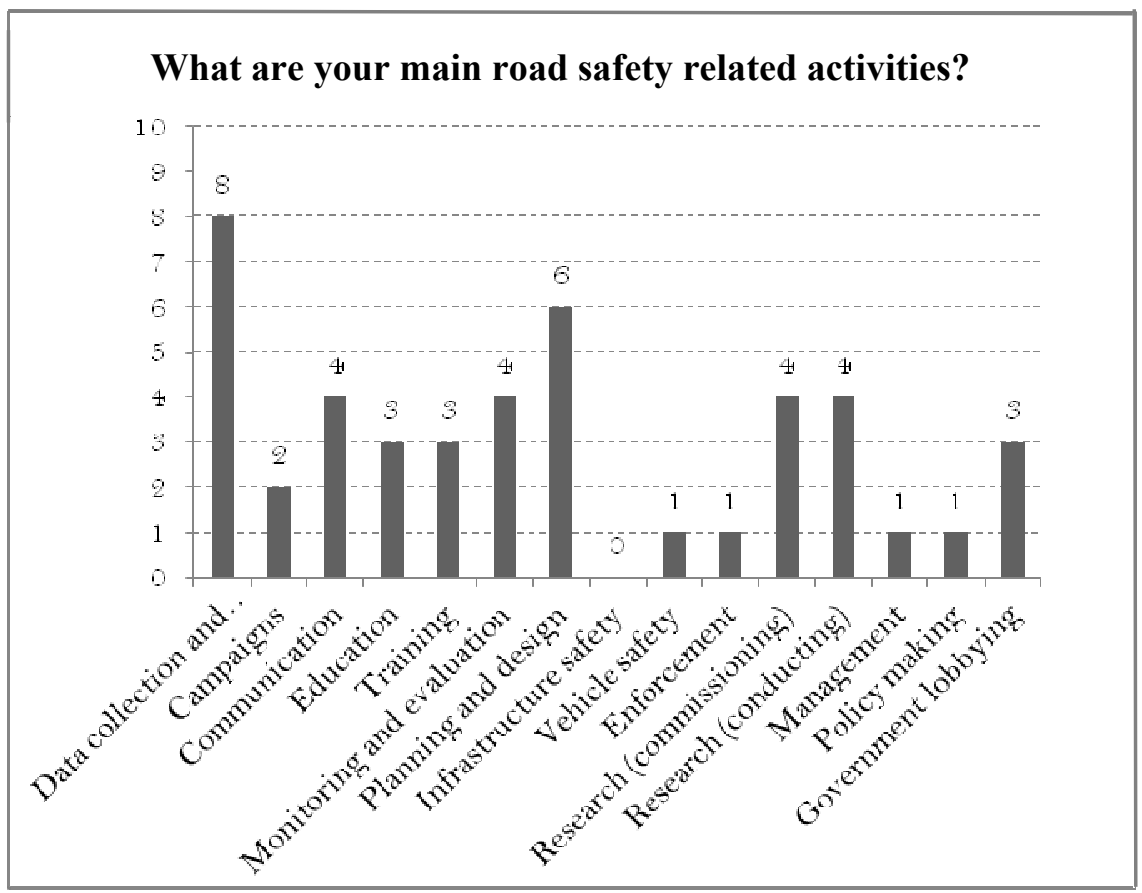

Figure 4: Main road safety related activities of the interviewed stakeholders.

Furthermore, the survey revealed that there is, among others, a need to increase the technicians' and public administrations' knowledge on road users' attitudes. Information on road users' behaviour and attitudes, which are of high priority (6) or medium priority (2) for most of the interviewed stakeholders, are indeed only partially (8) or not available (1) (figure 5). Carrying out wider and more systematic surveys on road users' can be very helpful to fill this gap.

\section{Opinion surveys applied to road safety analysis: some recommendations}

This paper has provided an overview of local-based surveys on road safety that took place in the territory of Brescia and in its surrounding area.

Road users' perceptions and opinions about transport and road safety should be very relevant to policy makers for understanding the needs of the public, the limitations of their policies and the potential support for new policies.

Among the main outcomes of the presented surveys, it is possible to place the attention on the fatalism that people often link to road accidents. Decision makers should apply surveys to improve the existing road safety strategies and to prioritise interventions. Interventions do not necessarily have to be infrastructural: educational measures and public awareness campaigns may have a high impact on road safety as well, even better if they are focused on the threats that road users perceive. 


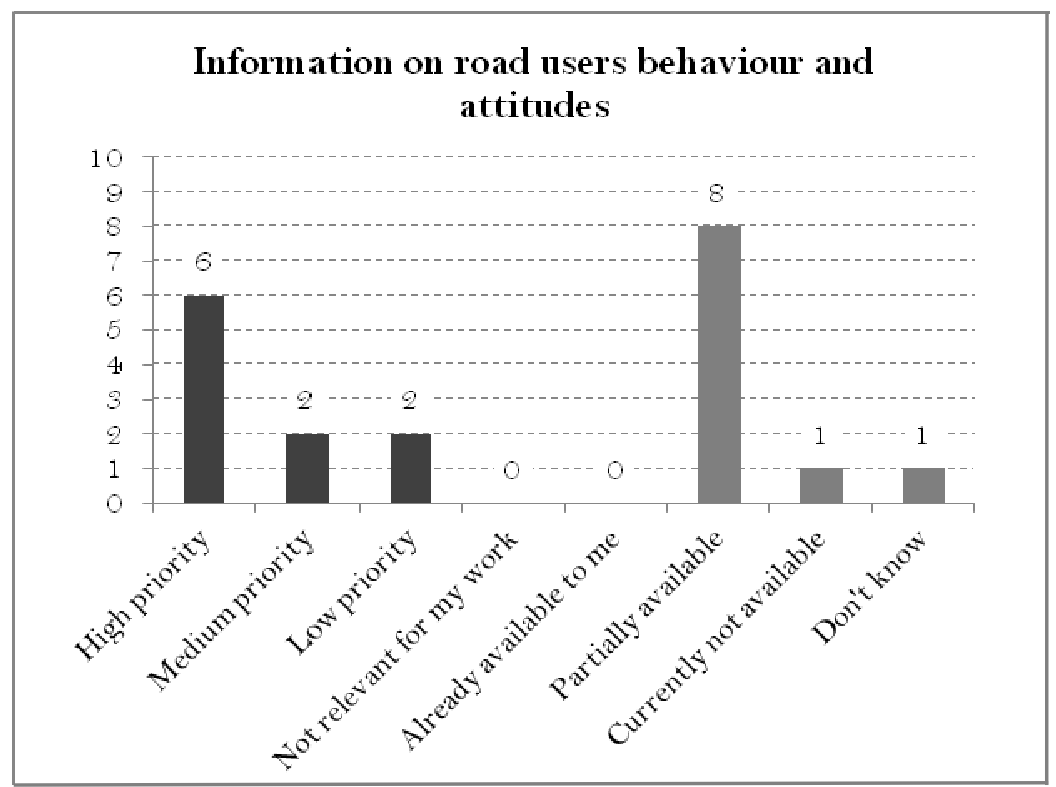

Figure 5: Priority and availability of information on road users' behaviour and attitudes.

Furthermore, some of the results have stressed the impact the accessibility issues have had on road safety. An improvement in the accessibility for vulnerable road users (e.g. with pedestrian paths, cycle lanes...), will increase the safety levels that they perceive.

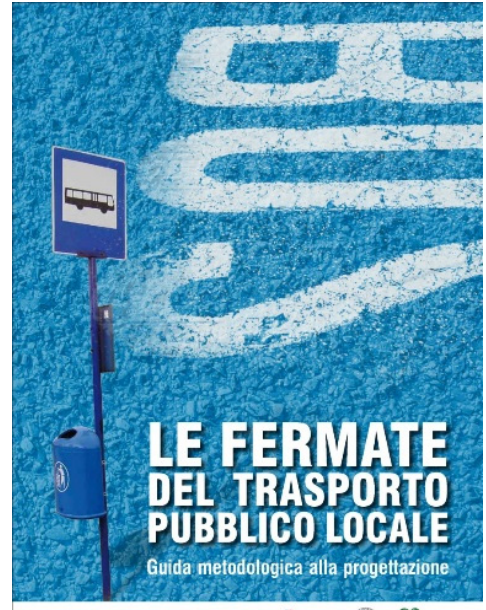

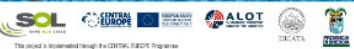
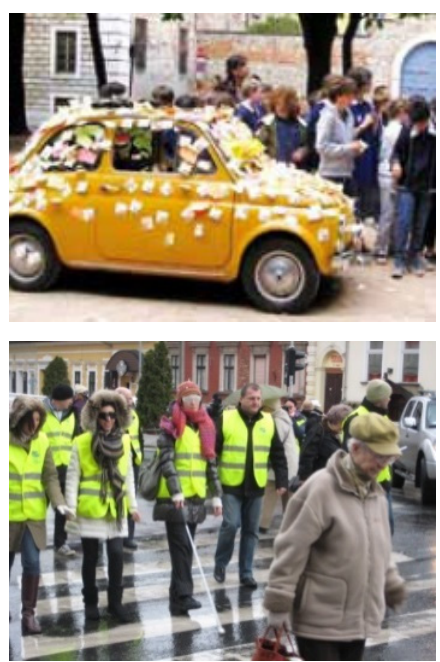

Figure 6: Examples of campaigns for technicians and road users. 
The surveys presented in this paragraph can be used as pilot-surveys, and similar approaches can be easily transferred and applied to other contexts, and to wider ones, including at a regional and the national level. Of course, in the latter case, the complexity of conducting the surveys will be higher, and will require more resources.

Although nowadays public opinion surveys are not frequently applied to road users, they can play a crucial role while dealing with road safety. Therefore, the use of surveys should increase, and the road users' perception of risk should be fully integrated with the diagnosis of road safety threats.

Lastly, which are the main methodological lessons learned by the developed pilot-surveys?

It is necessary to pay attention to every step of the survey methodology, and conduct the survey carefully, in order to obtain reliable results. During the first groundwork phase of setting the questionnaire, it must be reminded that surveys should generate quantitative responses (multi-choice) rather than qualitative (written option). The proposed surveys included few open questions (e.g. the students were asked if they had suggestions on how to improve public transport safety), but in the end they were scarcely considered during the elaboration phase, due to a lack of homogeneity in the responses. Furthermore, the dissemination of the questionnaires is a crucial phase. Nowadays there are some web-based tools that can be used to conduct surveys, generating a link where suitable people or institutions can complete the survey online. This would decrease the need for face-to-face surveys, but the process would be less controlled by the interviewer.

\section{Acknowledgements}

This research was supported by the project ROSEE - ROad Safety in South-East European regions, co-funded by the South East Europe Transnational Cooperation Program.

\section{References}

[1] Busi R., For a Safer City. A Friendlier City. And a More Beautiful City, in TeMALab journal of Mobility, Land Use and Environment, Selected Papers 2009, 3(2010): 39-46, 2010.

[2] Tira M. (editor), A methodological approach to national road safety policies, European Transport Safety Council - ETSC, Brussels, 2006.

[3] Tiboni M., Pianificazione urbanistica e sicurezza stradale. Dalla diagnostica alla definizione degli interventi per la mitigazione del rischio, Bios, Cosenza, 2004.

[4] Fleury D., Sécurité \& urbanisme. La prise en compte de la sécurité routière dans laménagement urbain, Presse de l'ENPC, Paris, 1998.

[5] Tiboni, M., Rossetti, S., Implementing a road safety review approach for existing bus stops, WIT Transactions on the Built Environment, 130, pp. 699-709, 2013. 
[6] Sapsford R., Survey Research, Second edition, SAGE Publications, London, 2007.

[7] Fowler F.J., Survey Research Methods, Fourth Edition, Applied social research methods series, SAGE Publications, California, 2009.

[8] Pronello C., Profili di mobilità ed accessibilità, in Castrignanò M., Colleoni M., Pronello C. (editors), Muoversi in città. Accessibilità e mobilità nella metropoli contemporanea, pp. 99-116, Franco Angeli, Milano, 2012.

[9] Albarracin D., Johnson B.T., Zanna M.P. (eds.), The handbook of attitudes, Lawrence Erlbaum, Mahwah, NJ, 2005.

[10] New Zealand, Ministry of Transport, Public attitudes to road safety, Results of the 2012 survey, Financial, Economic and Statistical Analysis Team of the Ministry of Transport, Wellington, 2012. 